\title{
LETTER \\ Feature Fusion for Blurring Detection in Image Forensics
}

\author{
BenJuan YANG ${ }^{\dagger, \dagger}$, Student Member and BenYong LIU ${ }^{\dagger a)}$, Nonmember
}

SUMMARY Artificial blurring is a typical operation in image forging. Most existing image forgery detection methods consider only one single feature of artificial blurring operation. In this manuscript, we propose to adopt feature fusion, with multifeatures for artificial blurring operation in image tampering, to improve the accuracy of forgery detection. First, three feature vectors that address the singular values of the gray image matrix, correlation coefficients for double blurring operation, and image quality metrics (IQM) are extracted and fused using principal component analysis (PCA), and then a support vector machine (SVM) classifier is trained using the fused feature extracted from training images or image patches containing artificial blurring operations. Finally, the same procedures of feature extraction and feature fusion are carried out on the suspected image or suspected image patch which is then classified, using the trained SVM, into forged or non-forged classes. Experimental results show the feasibility of the proposed method for image tampering feature fusion and forgery detection.

key words: image forensics, blurring detection, feature fusion, PCA, SVM

\section{Introduction}

Due to the rapid development and wide application of image editing software, a good forgery operation may leave no visual clues in the forged image. This complicates public security detection and judicial forensics. At the same time, however, it promotes the development of forgery detection technology [1], [2].

In image forging, blurring is a very commonly used operation. In [3], Hisiao applied a modified approach for blur estimation to detect blurred regions. In [4], Wang proposed an image forensics method using abnormity of local hue for blur detection. In [5], Sutcu described a blurring tamper detection technique based on regularity of wavelet transform coefficients. In [6], Zhou used mathematical morphology edge to exposing digital forgeries. In [7], Wang defined double blurring correlation, and used it to detect blurring operation. In [8], Zuo used subtraction of double blurring image to detect blurred regions. All of these methods depend on threshold values. Since there is no theoretical basis for the selection of threshold values, the threshold restricts the accuracy of algorithm.

Forgery detection is, in fact, a binary classification problem in the view of pattern recognition, so it may

Manuscript received October 16, 2013.

Manuscript revised February 19, 2014.

${ }^{\dagger}$ The authors are with Guizhou University, GuiYang, 550025 China.

${ }^{\dagger \dagger}$ The author is with Guizhou Normal University, GuiYang, 550002 China.

a)E-mail: csc.byliu@gzu.edu.cn

DOI: 10.1587/transinf.E97.D.1690 be solved by pattern recognition approaches, wherein feature extraction, feature fusion and classification are three paramount stages. In this manuscript, we propose to adopt feature fusion, with multifeatures of artificial blurring operation, to improve the accuracy of forgery detection, and adopt support vector machine (SVM) to avoid threshold calculation. Firstly, three types of feature vectors are extracted and fused using principal component analysis (PCA), and then an SVM classifier is trained using the fused feature extracted from original images and forged images containing artificial blurring operations. Finally, for a suspected image or a suspected image patch, the same procedure is carried out to detect artificial blurring operation.

\section{Proposed Method for Blurring Detection}

The proposed image forgery detection method is systematically depicted in Fig. 1. In the training step, three types of feature vectors introduced in the sequel are extracted from original images and forged images. The next step is feature fusion using PCA described in 2.2. After that, a classifier is trained to represent the sample features. The radial basis function (RBF) is chosen as the kernel function of SVM. After optimum seeking in the training process, cross validation is used to determine the parameters in RBF. Finally, the same type of feature sets are extracted from testing image or image patch and fused into a multifeature vector, and then fed into the trained classifier to get the detection result.

\subsection{Feature Extraction}

For blurring tampering in image forensics, many scholars defined some measurement factors as follows, in which $I$ is an $M \times N$ image or image patch, and $I^{\prime}$ is the degraded

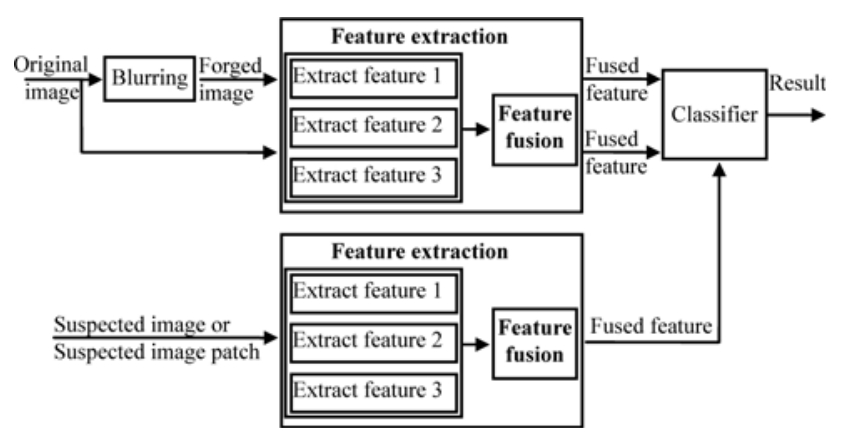

Fig. 1 Image forgery detection system based on feature fusion. 
version of $I$.

a. Singular Values Decomposition (SVD) Feature

Since the linear correlation of image pixels is increased by blurring operation, the singular values of a gray image matrix with high indexes tend to decrease, i.e., the energy of high indexed singular values is significantly decreased. According to the changes in the singular values which brought by blurring operation, we define three measurement factors as follows, which are denoted respectively by $C D 1, C D 2$, and $C D 3$.

First, let

$$
\begin{aligned}
& I=U \Sigma V^{*}, \quad S=\operatorname{diag}(\Sigma), \\
& m=\min (M, N), \quad n=\lfloor m / 2\rfloor, \\
& p(k)=S(k) / \sum_{i=1}^{m} S(i), \quad k=1,2, \ldots, m .
\end{aligned}
$$

And then

$$
\begin{aligned}
C D 1 & =\sum_{i=n+1}^{m} P(i) / n, \\
C D 2 & =\sum_{i=1}^{m} u(S(i)) / n, \quad u(k)= \begin{cases}1, & k<2 \\
0, & k \geq 2\end{cases}
\end{aligned}
$$

And let $T 1=\{C D 1, C D 2, C D 3\}$.

\section{b. Double Blurring Correlation Feature}

In [7], Wang defined double blurring correlation and proved that the double blurring correlation of blurred image is more salient than that of original image. A feature measurement factor is defined in [7] for double blurring correlation feature extraction as follows, which is denoted by $C D 4$.

$$
C D 4=\rho_{\text {corr }}\left(|\ln (F(I))|,\left|\ln \left(F\left(I^{\prime}\right)\right)\right|\right) .
$$

Where $F$ is Fourier transform. Let $T_{2}=\{C D 4\}$.

\section{c. Image Quality Metrics (IQM) Feature}

Since the quality of images may be degraded by blurring operation, IQM is adopted to detect artificial blurring operation traces [8]. However, the IQM defined in [8] is essentially equivalent to Mean Absolute Error, and thus we adopt the IQM defined in [9] to create five measurement factors, and they are Mean Absolute Error, Mean Square Error, Minkowsky Measure, Content Structure Correlation, and Normalized Cross-correlation which are denoted by $C D 5$ to $C D 9$, respectively, with

$$
\begin{aligned}
& C D 5=\sum_{i=1}^{M} \sum_{j=1}^{N}\left|I(i, j)-I^{\prime}(i, j)\right| / M N, \\
& C D 6=\left(\sum_{i=1}^{M} \sum_{j=1}^{N}\left|I(i, j)-I^{\prime}(i, j)\right|^{2} / M N\right)^{\frac{1}{2}}, \\
& C D 7=\max \left(\left|I(i, j)-I^{\prime}(i, j)\right|\right), \\
& C D 8=\sum_{i=1}^{M} \sum_{j=1}^{N} I(i, j)^{2} / \sum_{i=1}^{M} \sum_{j=1}^{N} I^{\prime}(i, j)^{2},
\end{aligned}
$$

$$
C D 9=\sum_{i=1}^{M} \sum_{j=1}^{N} I(i, j) I^{\prime}(i, j) / \sum_{i=1}^{M} \sum_{j=1}^{N} I(i, j)^{2} .
$$

And let $T_{3}=\{C D 5, C D 6, C D 7, C D 8, C D 9\}$.

\subsection{Feature Fusion with PCA}

PCA is a powerful technique for extracting structures from possibly high-dimensional data sets [10]. From the view point of mathematics, PCA is an orthogonal transformation of the coordinate system in which the data are described. The new coordinate values, by which the data are represented, are called principal components. It is often the case that a small number of principal components are sufficient to account for the main structure embedded in data. Data are projected into the subspace spanned by the first $k$ eigenvectors of the covariance matrix of the training set, and the choice of $k$ usually based on contribution rate $c L$. In this paper, we use PCA to fuse feature in three ways.

\section{a. Scheme 1}

Directly combine the aforementioned three single features into a multifeature, and then adopt PCA to form a fused multifeature vector. The flow chart of Scheme 1 is shown in Fig. 2, where $T_{i}(i=1,2,3)$ denote the SVD feature, the double blurring correlation feature and the IQM feature, respectively, while $T=\left\{T_{1}, T_{2}, T_{3}\right\}$ is a directly combined multifeature, and $T^{\prime}$ is a fused feature vector from $T$ by PCA.

\section{b. Scheme 2}

The flow chart of Scheme 2 is shown in Fig. 3, where $T_{i}$ $(i=1,2,3)$ denote the same features described above, while $T_{i}^{\prime}(i=1,2,3)$ is a fused feature from $T_{i}(i=1,2,3)$ by PCA, and $T^{\prime}=\left\{T_{1}^{\prime}, T_{2}^{\prime}, T_{3}^{\prime}\right\}$.

\section{c. Scheme 3}

The flow chart of Scheme 3 is shown in Fig. 4, where $T_{i}$ $(i=1,2,3)$ denote the same features as above, while $T_{i}^{\prime}$

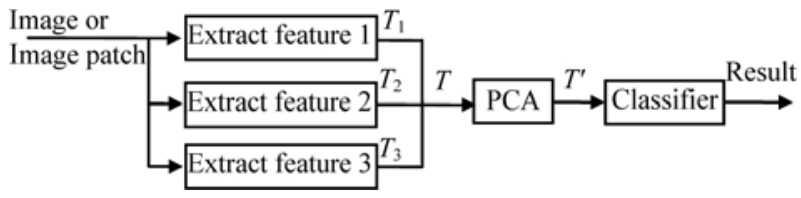

Fig. 2 Flow chart of feature fusion in Scheme 1.

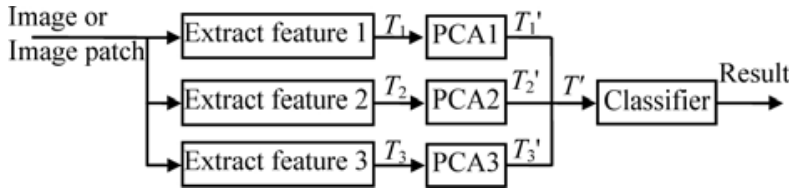

Fig. 3 Flow chart of feature fusion in Scheme 2.

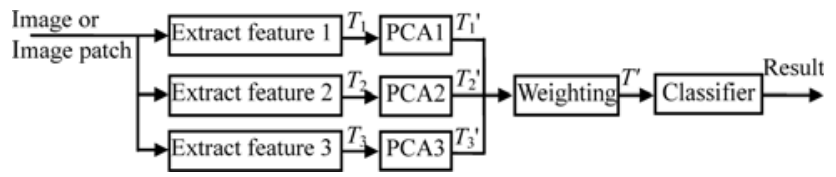

Fig. 4 Flow chart of feature fusion in Scheme 3. 
$(i=1,2,3)$ is a fused feature from $T_{i}(i=1,2,3)$ by PCA, and $T^{\prime}=\left\{\omega_{1} T_{1}^{\prime}, \omega_{2} T_{2}^{\prime}, \omega_{3} T_{3}^{\prime}\right\}$ is a weighted fusion feature, where $\omega_{1}+\omega_{2}+\omega_{3}=1$.

\section{Experiment}

In our experiments, the sample set is consisted of some original images and the forged versions, wherein 183 authentic images in the Columbia Color Image Splicing Detection Evaluation Dataset are taken as the original images, and they are blurred in MATLAB using Gaussian blurring kernel with size $5 \times 5$ and kernel width $\sigma=2$, and then taken as forged images. To evaluate the performance of the proposed method, each correct detection rate is the average of those of 20 runs. In each run, the training samples are randomly selected from the image dataset to train the classifier.

\subsection{Experiment 1}

We select the training sample size to be $1 / 4,1 / 2$, and $3 / 4$ of images to compare the correct detection rates of the algorithms with one single feature and directly combined multifeature. The remaining images are used in testing. The result of Experiment 1 is shown in Fig. 5.

Compared to the prior arts in [7], [8], using SVD feature defined in this manuscript we achieve average correct detection rate of $91 \%$, which is better than the double blurring correlation feature in [7] and the IQM feature in [8], wherein the rates are $88 \%$ and $75 \%$ respectively. It is observed that by directly combining these three feature sets, we achieve $93 \%$, which is far better.

\subsection{Experiment 2}

We select the training sample size to be $1 / 4,1 / 2$, and $3 / 4$ of images to compare the correct detection rates of the algorithms with directly combined multifeature and fused feature. The result of Experiment 2 is shown in Fig. 6. It shows that feature fusion is better than direct combination, and thus the effect of Scheme 3 is best. So, in the subsequent, we will adopt feature fusion Scheme 3. In our experiment, $c L=75 \%, c L_{1}=100 \%, c L_{2}=100 \%, c L_{3}=25 \%$,

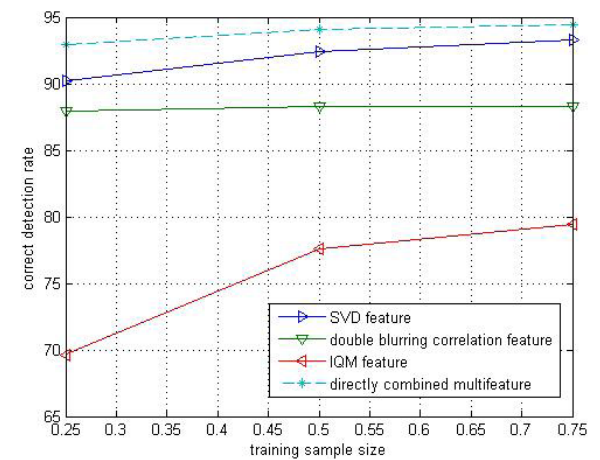

Fig. 5 Correct detection rates of the algorithms with one single feature and directly combined multifeature. $\omega_{1}=0.55, \omega_{2}=0.35, \omega_{3}=0.1$.

\subsection{Experiment 3}

We use Photo-shop to blur a portion of an original image with different parameter values shown in Table 1, and obtain the forged images as shown in Figs. 7 (a1)-(a5). Then we use the methods in [7], [8], with directly combined multifeature and feature fusion Scheme 3 to test these images. The results are shown in Figs. 7 (b)-(e). Experimental results show that one single feature alone is effective, but not good, that is, there are many false-alarms. If these features are combined, the result is better. But the best result is achieved by feature fusion Scheme 3 .

\section{Conclusion}

In this paper, we proposed an effective algorithm for detecting the traces of artificially blurring in an image in the absence of any digital watermark or signature. Unlike existing methods, it avoids threshold calculation and improves performance accuracy. We extract three features to address artificial blurring operation, and then use PCA to fuse them into a multifeature vector. An SVM classifier is trained using the fused feature vector and applied to detect possible blurring operation in suspect images. Experimental results show the feasibility of the proposed method for image tampering feature fusion and forgery detection. Since our algorithm focuses on detecting artificial blurring operation, it may be ineffective for detecting other forms of digital tampering. That problem will be tackled in future work.

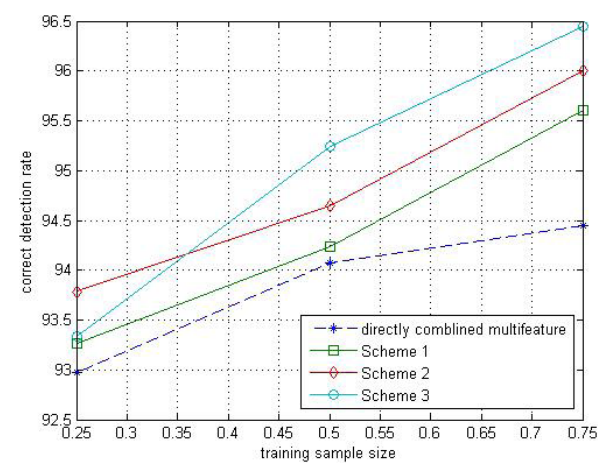

Fig. 6 Correct detection rates of the algorithms with directly combined multifeature and fused feature.

Table 1 Different blurring operations and parameter setting.

\begin{tabular}{ll}
\hline Blur filter & Radius/pixels \\
\hline (a1) Motion Blur & 10 \\
(a2) Shape Blur & 5 \\
\hline (a3) Box Blur & 2 \\
\hline (a4) Lens Blur & 6 \\
\hline (a5) Gaussian Blur & 1 \\
\hline
\end{tabular}




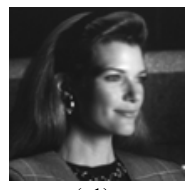

(a1)

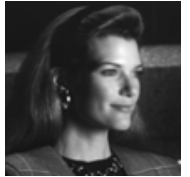

(a2)

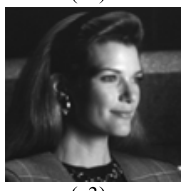

(a3)

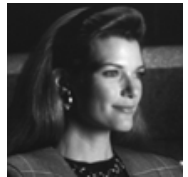

(a4)

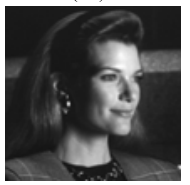

(a5)

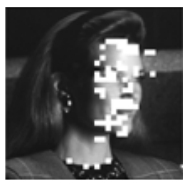

(b1)

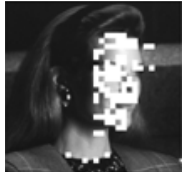

(b2)

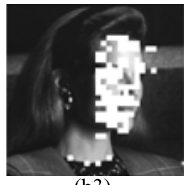

(b3)

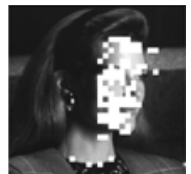

(b4)

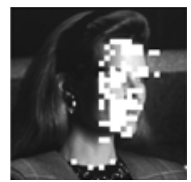

(b5)

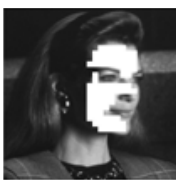

(c1)

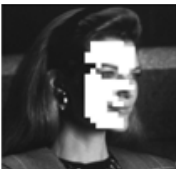

(c2)

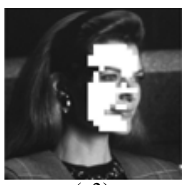

(c3)

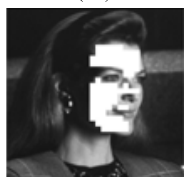

(c4)

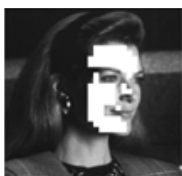

(c5)

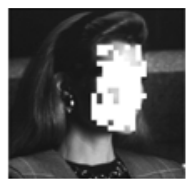

(d1)

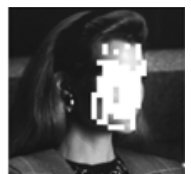

(d2)

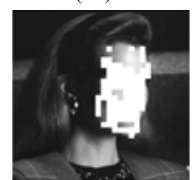

(d3)

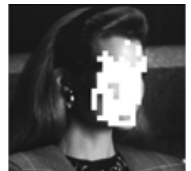

(d4)

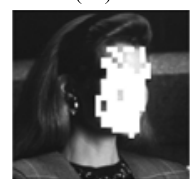

(d5)

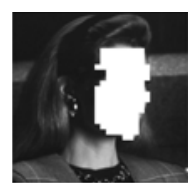

(e1)

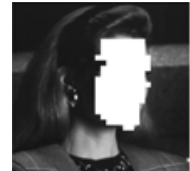

(e2)

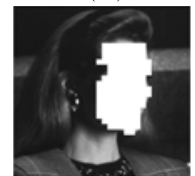

(e3)

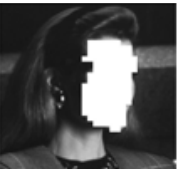

(e4)

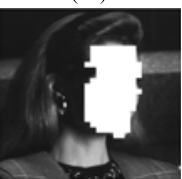

(e5)

Fig. 7 Artificially blurred images with different operations and parameters (First Column), forgery detection results using double blurring correlation feature in [7] (Second Column), IQM feature in [8] (Third Column), directly combined multifeature (Fourth Column), and feature fusion Scheme 3 (Fifth Column).

\section{Acknowledgments}

This work was supported in part by grants of the Natural Science Foundation of China (60862003), Project of Int'l S\&T Cooperation Program of China (2009DFR10530), The Ph.D. Programs Foundation of Ministry of Education of China (20095201110002), Industrial-Key-ProblemTackling Program of Guizhou S\&T Bureau (Qian Ke He GY Word (2010) 3054), and Guizhou Science and Technology Fund (Qian Ke He J Word (2012) 2272).

\section{References}

[1] H. Farid, "Image forgery detection," IEEE Trans. Signal Process., vol.26, no.2, pp.16-25, 2009.

[2] W.Q. Luo, Z.H. Qu, F. Pan, and J.W. Huang, "A survey of passive technology for digital image forensics," Frontiers of Computer Science in China, vol.2, no.1, pp.166-179, 2007.

[3] D.Y. Hsiao and S.C. Pei, "Detecting digital tampering by blur estimation," Proc. IEEE 1st International Workshop on Systematic Approaches to Digital Forensic Engineering, pp.264-278, Piscataway,
USA, 2005.

[4] B. Wang, L.L. Sun, X.W. Kong, and X.G. You, "Image forensics technology using abnormity of local hue for blur detection," Acta Electronica Sinica, vol.34, no.12A, pp.2451-2454, 2006.

[5] Y. Sutcu, B. Coskun, H.T. Sencar, and N. Memon, "Tamper detection based on regularity of wavelet transform coefficients," Proc. IEEE International Conference on Image Processing, pp.397-400, San Antonio, USA, 2007.

[6] L.N. Zhou, D.M. Wang, Y.B. Guo, and Y.X. Yang, "Exposing digital forgeries by detecting image blurred mathematical morphology edge," Acta Electronica Sinica, vol.36, no.6, pp.1047-1051, 2008.

[7] W. Wang and Y. Fang, "Blind separation of single channel permuted image based on double blur correlation," J. Applied Sciences, vol.29, no.2, pp.169-175, 2011.

[8] J.X. Zuo and B.Y. Liu, "Detection for tampering operations in a forged image," J. Image and Graphics, vol.17, no.11, pp.1367-1375, 2012.

[9] C.H. Zhou, Y.J. Hu, and L.L. Tang, "Study on performance of typical source camera classification algorithms," J. Computer Applications, vol.31, no.4, pp.1133-1137, 2011.

[10] R.O. Duda, P.E. Hart, and D.G. Stork, Pattern classification, 2nd ed., Wiley, New York, USA, 2001.

[11] V.N. Vapnik, Statistical learning theory, Wiley, New York, USA, 1998. 